Preface

https://doi.org/10.52825/bis.v1i.70

(C) Authors. This work is licensed under a Creative Commons Attribution 4.0 International License

Published: 02 July 2021

\title{
24th International Conference on Business Information Systems
}

\author{
Preface \\ Witold Abramowicz ${ }^{1 \text { [https://orcid.org/0000-0001-5464-9698] }}$ and Sören Auer ${ }^{2[\text { https://orcid.org/0000-0002-0698-2864] }}$ \\ ${ }^{1}$ Poznań University of Economics and Business, Poland \\ ${ }^{2}$ Technische Informationsbibliothek, Germany
}

The theme of the 24th BIS conference was Enterprise Knowledge and Data Spaces. Both concepts are relevant for data organization and reuse. One of the contemporary ways to represent knowledge in the enterprises are enterprise knowledge graphs or just knowledge graphs. They are a flexible way to represent interlinked information about virtually anything. From the modelling point of view they are graphs consisting of concepts, properties, and entity descriptions. So, we distinguish here schema data, instance data, and metadata. What is important is that they are fully compliant to FAIR Data Principles. The principles characterize the desired features of digital assets: Findable, Accessible, Interoperable, Reusable.

Data spaces are more about information technology architecture. They are considered a virtual space for safeguarding data where data remains with the data owner and it is first shared with trusted business partners. The focus is on the information model for describing data assets and also providing the standardized interface for interoperability. More often than in the case of enterprise knowledge we emphasize the economic valuation of data, including pricing of data transactions. It is noteworthy that data spaces are considered a core of the implementation of the European Strategy on Data. It foreseen to regulate the flow of data within European Union and across sectors and is also based on the FAIR principles. As the ultimate goal of FAIR is to optimise the reuse of data we expect papers covering all aspects of data collection, standardization, integration, exchange, reuse, and valuation.

The 24th edition of International Conference on Business Information Systems was held in Hannover, Germany. Due to the COVID-19 pandemic, the conference was held as an online event. Since its first edition in 1997, the BIS conference became a well-respected event and it gathered a wide and active community of researchers and business practitioners.

The BIS 2021 proceedings includes 32 articles divided into 7 parts that reflect main areas of interests of BIS community. Those are: Big Data, Smart Infrastructure, Knowledge Graphs, Artificial Intelligence, Social Media, Applications and ICT Projects. Authors submitted 93 papers total thus the acceptance rate was $34 \%$.

The Program Committee consisted of 105 members from 30 countries, who carefully evaluated all the submitted papers.

We would like to sincerely thank all people who are involved in the BIS community. The reviewers, who dedicate their time and prepare insightful comments. Keynote Speakers, for their interesting presentations that started a vivid discussion among the conference participants. Last but not least, we would like to thank all authors who submitted their papers. June, 2021, Witold Abramowicz \& Sören Auer 